\title{
Corrigendum
}

Diabetes Metab J 2020;44:783

https://doi.org/10.4093/dmj.2020.0245

pISSN 2233-6079 • eISSN 2233-6087

DIABET\&S \& METABOLISM JOURNAL

\section{Associations among Obesity Degree, Glycemic Status, and Risk of Heart Failure in 9,720,220 Korean Adults}

Eun Jung Rhee ${ }^{1}$, Hyemi Kwon ${ }^{1}$, Se Eun Park ${ }^{1}$, Kyung Do Han², Yong Gyu Park², Yang Hyun Kim³ , Won Young Lee ${ }^{1}$

${ }^{1}$ Division of Endocrinology and Metabolism, Department of Internal Medicine, Kangbuk Samsung Hospital, Sungkyunkwan University School of Medicine, Seoul,

${ }^{2}$ Department of Biostatistics, Biomedicine \& Health Sciences, College of Medicine, The Catholic University, Seoul,

${ }^{3}$ Department of Family Medicine, Korea University Anam Hospital, Korea University College of Medicine, Seoul, Korea

Diabetes Metab J 2020;44:592-601. https://doi.org/10.4093/dmj.2019.0104

In this Article, Model 3 in Table 2 is unnecessarily added. It was deleted from the corrected Table as follows.

Table 2. Risk for heart failure according to obesity degree

\begin{tabular}{|c|c|c|c|c|c|c|c|}
\hline Variable & $\begin{array}{c}\text { No. of } \\
\text { subjects }\end{array}$ & $\begin{array}{l}\text { No. of } \\
\text { event }\end{array}$ & $\begin{array}{c}\text { Incidence } \\
\text { rate, } / 1,000 \\
\text { person-years }\end{array}$ & Model 1 & $P$ value & Model 2 & $P$ value \\
\hline BMI levels, $\mathrm{kg} / \mathrm{m}^{2}$ & - & & & & & & \\
\hline$<18.5$ & 372,523 & 3,855 & 1.67 & $1.47(1.42-1.52)$ & $<0.001$ & $1.39(1.35-1.44)$ & $<0.001$ \\
\hline $18.5-22.9$ & $3,848,812$ & 28,968 & 1.20 & 1 (reference) & - & 1 (reference) & - \\
\hline $23.0-24.9$ & $2,406,196$ & 20,934 & 1.38 & $1.60(1.04-1.08)$ & $<0.001$ & $0.91(0.90-0.93)$ & $<0.001$ \\
\hline $25.0-29.9$ & $2,762,426$ & 28,319 & 1.63 & $1.20(1.20-1.22)$ & $<0.001$ & $0.93(0.92-0.95)$ & $<0.001$ \\
\hline$\geq 30$ & 330,263 & 4,249 & 2.05 & $1.62(1.57-1.68)$ & $<0.001$ & $1.15(1.11-1.18)$ & $<0.001$ \\
\hline$P$ for trend & & & & $<0.001$ & & $<0.001$ & \\
\hline \multicolumn{8}{|l|}{ WC levels, men/women } \\
\hline$<80.0 / 75.0 \mathrm{~cm}$ & $3,611,228$ & 19,159 & 0.84 & $0.72(0.71-0.74)$ & $<0.001$ & $0.91(0.89-0.93)$ & $<0.001$ \\
\hline $80.0 / 75.0-84.9 / 79.9 \mathrm{~cm}$ & $2,314,707$ & 18,018 & 1.24 & $0.85(0.83-0.87)$ & $<0.001$ & $0.94(0.92-0.96)$ & $<0.001$ \\
\hline $85.0 / 80.0-89.9 / 84.9 \mathrm{~cm}$ & $1,918,487$ & 19,988 & 1.66 & 1 (reference) & - & 1 (reference) & - \\
\hline $90.0 / 85.0-94.9 / 89.9 \mathrm{~cm}$ & $1,117,820$ & 14,766 & 2.10 & $1.16(1.14-1.19)$ & $<0.001$ & $1.04(1.02-1.07)$ & $<0.001$ \\
\hline 95.0/90.0-99.9/94.9 cm & 493,564 & 8,526 & 2.75 & $1.33(1.29-1.36)$ & $<0.001$ & $1.16(1.13,1.19)$ & $<0.001$ \\
\hline$\geq 100.0 / 95.0 \mathrm{~cm}$ & 264,414 & 5,868 & 3.55 & $2.00(1.94-2.05)$ & $<0.001$ & $1.48(1.43-1.52)$ & $<0.001$ \\
\hline$P$ for trend & & & & $<0.001$ & & $<0.001$ & \\
\hline
\end{tabular}

Values are presented as hazard ratio (95\% confidence interval). Model 1 adjusted for age and sex; Model 2 adjusted for age, sex, smoking, alcohol drinking, regular exercise, low income, hypertension, dyslipidemia, and chronic kidney disease.

$\mathrm{BMI}$, body mass index; WC, waist circumference.

This Table has been corrected in the online versions.

We apologize for any inconvenience that this may have caused.

This is an Open Access article distributed under the terms of the Creative Commons Attribution Non-Commercial License (https://creativecommons.org/licenses/by-nc/4.0/) which permits unrestricted non-commercial use, distribution, and reproduction in any medium, provided the original work is properly cited. 